\title{
Experimental and numerical investigation of eigenfrequencies of rectangular plates, interacting with a fluid
}

\author{
Sergey Bochkarev ${ }^{1, \star}$, Alexander Kamenskikh ${ }^{2}$, and Sergey Lekomtsev ${ }^{1}$ \\ ${ }^{1}$ Institute of Continuous Media Mechanics RAS, 1 Acad. Korolev str., Perm, 614013, Russian Federation \\ ${ }^{2}$ Perm National Research Polytechnic University, 29 Komsomolsky ave., Perm, 614990, Russian Federation
}

\begin{abstract}
The method of laser vibrometry was applied to investigate the vibrations of elastic plates interacting with a liquid for different variants of plate clamping. The obtained results are used to verify the developed finiteelement algorithm designed to solve the problem of spatial vibrations of one or two parallel rectangular plates interacting with a quiescent viscous fluid. The results of calculations of the natural frequencies and damping decrement obtained under different boundary conditions and the height of the fluid layer are discussed. It has been demonstrated that for the configurations considered, viscosity of the liquid has a significant effect on the damping decrement, in contrast to frequencies of vibrations.
\end{abstract}

\section{Introduction}

Interest in the problems of natural vibrations of plates interacting with a fluid is due to the availability of various technical applications with strict requirements for safety and performance characteristics of structures. Typical examples include trash racks of hydroelectric plants [1] or parallel-plate fuel assemblies of power plants. Recent literature includes numerous papers, in which the influence of various factors on the natural vibrational frequencies of plates interacting with a fluid is analyzed. Detailed chronology and review of relevant papers are presented in articles [2,3].

Experimental studies on this topic are represented by a limited number of works. In particular, horizontal circular plates completely immersed in a liquid are studied in [2], and rectangular ones - in [3,4]. One of the main results of these studies is the conclusion that natural frequencies of vibrations tend to an asymptotic value when the characteristic size of the structure exceeds the height of the fluid layer. The setup developed in [3] allows one to change the position of the plate and to examine partially submerged vertical plates. In the recently published paper [5], the natural frequencies and mode shapes of a horizontal plate, overlaid with a fluid layer have been investigated theoretically and experimentally. Here, measurements were made in two ways. In the first case, a sensor based on polyvinylidene difluoride (PVDF) was used, and in the second case, measurements were taken using the amplitude-fluctuation electronic speckle pattern interferometry (AF-ESPI) method.

In the dynamics of thin-walled structures, along with the frequencies and modes of vibrations, it is of practical interest to study the damping characteristics of the system.

\footnotetext{
^e-mail: bochkarev@icmm.ru
}

In the case of hydroelastic interaction, they can also be determined by viscosity properties of the liquid. In the majority of works, the authors used model of an ideal fluid, in which damping was not taken into account. In this connection, the development of a numerical algorithm for solving the problem of spatial vibrations of plates interacting with a quiescent viscous fluid, and its verification with the help of experiment, are the problems of direct interest.

\section{Mathematical formulation}

We consider a prismatic tank of length $a$, width $b$, and height $H$, containing fluid. It has perfectly rigid walls and is bounded from below and above (or only from above) by elastic rectangular plates of thickness $h$. Let the system be set in motion under the action of some perturbations. First, we formulate the constitutive relations for the case of one plate.

The vortex-free perturbed motion of a viscous fluid is described in the framework of the acoustic approximation in terms of the perturbed velocity potential $\phi[6]$

$$
\frac{\partial^{2} \phi}{\partial t^{2}}=\left(c^{2}+\frac{4 v}{3} \frac{\partial}{\partial t}\right) \nabla^{2} \phi
$$

where $c, v$ are the sound velocity and kinematic viscosity of the fluid.

The components of the stress tensor of the fluid are determined by relation [6]

$$
\sigma_{i j}^{f}=-\left(-\rho_{f} \frac{\partial \phi}{\partial t}+2 \mu \nabla^{2} \phi\right) \delta_{i j}+2 \mu \frac{\partial^{2} \phi}{\partial x_{i} \partial x_{j}}
$$

where $\mu$ and $\rho_{f}$ are the coefficients of viscosity and fluid density, $\delta_{i j}$ is the Kronecker symbol, $i, j$ take the values $x, y, z$. 
On the boundary of the elastic plate and fluid $S_{\sigma}$ the condition of equality of velocities and surface forces are prescribed

$$
\begin{gathered}
\dot{\mathbf{u}}=\mathbf{v}, \\
\sigma_{i j}^{f} n_{j}=-\sigma_{i j}^{s} n_{j},
\end{gathered}
$$

where $\mathbf{u}=\{u, v, w\}, \mathbf{v}=\left\{v_{x}, v_{y}, v_{z}\right\}$ are the vectors of the plate displacement and fluid velocity, $u, v, w$ are the displacements of points at the midsurface of the plate in the direction of the corresponding axes of the coordinate system $(x, y, z), \sigma_{i j}^{s}, \sigma_{i j}^{f}$ are the components of stress tensors of the plate and the fluid, $n_{j}$ are the vector components of the unit normal $\mathbf{n}=\left\{n_{x}, n_{y}, n_{z}\right\}$ to the surface $S_{\sigma}$.

In the case of small displacements, the vector of the normal $\mathbf{n}$ to the surface $S_{\sigma}$ slightly deviates from the $O z$ axis of the Cartesian coordinate system. Then, the boundary conditions (3), (4), with account of (2) take the following form:

$$
\begin{gathered}
\frac{\partial \phi}{\partial x}=\dot{u}, \quad \frac{\partial \phi}{\partial y}=\dot{v}, \quad \frac{\partial \phi}{\partial z}=\dot{w} . \\
t_{x}=-2 \mu \frac{\partial^{2} \phi}{\partial x \partial z}, \quad t_{y}=-2 \mu \frac{\partial^{2} \phi}{\partial y \partial z} \\
t_{z}=-\rho_{f} \frac{\partial \phi}{\partial t}+2 \mu\left(\nabla^{2} \phi-\frac{\partial^{2} \phi}{\partial z^{2}}\right) .
\end{gathered}
$$

Here $\mathbf{t}=\left\{t_{x}, t_{y}, t_{z}\right\}$ is the vector of surface forces of the plate.

At the interface between the fluid and the rigid wall $S_{w}$ we set the following condition

$$
v_{n}=\partial \phi / \partial n=0 .
$$

The small deformations of the elastic plate resulting from the hydroelastic interaction are determined by the first-order shear deformation plate theory [7]

$$
\begin{gathered}
\boldsymbol{\varepsilon}=\left\{\varepsilon_{x x}, \varepsilon_{y y}, \gamma_{x y}, \gamma_{x z}, \gamma_{y z}\right\}^{\mathrm{T}}=\tilde{\boldsymbol{\varepsilon}}+z \tilde{\mathbf{k}}, \\
\tilde{\boldsymbol{\varepsilon}}=\left\{\frac{\partial u}{\partial x}, \frac{\partial v}{\partial y}, \frac{\partial u}{\partial y}+\frac{\partial v}{\partial x}, \frac{\partial w}{\partial x}+\theta_{x}, \frac{\partial w}{\partial y}+\theta_{y}\right\}^{\mathrm{T}}, \\
\tilde{\mathbf{k}}=\left\{\frac{\partial \theta_{x}}{\partial x}, \frac{\partial \theta_{y}}{\partial y}, \frac{\partial \theta_{x}}{\partial y}+\frac{\partial \theta_{y}}{\partial x}, 0,0\right\}^{\mathrm{T}},
\end{gathered}
$$

where $\theta_{x}$ and $\theta_{y}$ are the angles of rotation about the $y$ and $x$-axes.

The physical equation that establishes the relation between the force and moment vector $\mathbf{T}$ and the strain vector $\varepsilon$, is written in the matrix form as

$$
\mathbf{T}=\left\{N_{x x}, N_{y y}, N_{x y}, M_{x x}, M_{y y}, M_{x y}, Q_{x}, Q_{y}\right\}^{\mathrm{T}}=\mathbf{D} \boldsymbol{\varepsilon} .
$$

The elements of the matrix $\mathbf{D}$ for isotropic material are determined in terms of the elastic modulus $E$, the Poisson's ratio $v$ and the shear modulus in the known manner.

A mathematical formulation of the problem of plate dynamics is based on the variational principle of virtual displacements, which includes terms that take into account the work of hydrodynamic pressure and inertia forces. It can be written in the matrix form as

$$
\begin{gathered}
\int_{S_{s}} \delta \boldsymbol{\varepsilon}^{\mathrm{T}} \mathbf{D} \boldsymbol{\varepsilon} d S+\int_{V_{s}} \rho_{s} \delta \mathbf{d}^{\mathrm{T}} \ddot{\mathbf{d}} d V-\int_{S_{\sigma}} \delta \mathbf{d}^{\mathrm{T}} \mathbf{p} d S=0, \\
\mathbf{p}=\left\{t_{x}, t_{y}, t_{z}, 0,0\right\}^{\mathrm{T}},
\end{gathered}
$$

where $\rho_{s}$ is the density of the plate, $\mathbf{d}$ and $\mathbf{p}$ are the vectors of the generalized displacements and surface loads, $S_{s}$ is the surface that bounds the volume of the plate $V_{s}$. Dots signify the first and second time derivatives.

\section{Numerical formulation}

When solving the problem of fluid dynamics by the Bubnov-Galerkin method, the formulation involving the wave equation (1) and boundary conditions (5), (7), is transformed to a weak form. The subsequent application of the standard procedures of the finite element method (FEM) leads to a matrix expression

$$
\begin{gathered}
\mathbf{M}_{f} \ddot{\mathbf{f}}+\mathbf{C}_{f}^{v} \dot{\mathbf{f}}+\mathbf{K}_{f} \mathbf{f}+\mathbf{M}_{f s}^{v} \ddot{\mathbf{w}}+\mathbf{C}_{f s} \dot{\mathbf{w}}=0, \\
\mathbf{M}_{f}=\sum_{m_{f}} \int_{V_{f}} \frac{\mathbf{F}^{\mathrm{T}} \mathbf{F}}{c^{2}} d V, \mathbf{C}_{f}^{v}=\sum_{m_{f}} \int_{V_{f}} \frac{4 v \Phi}{3 c^{2}} d V, \\
\mathbf{K}_{f}=\sum_{m_{f}} \int_{V_{f}} \Phi d V, \mathbf{M}_{f s}^{v}=-\sum_{m_{\sigma}} \int_{S_{\sigma}} \frac{4 v \mathbf{F}^{\mathrm{T}} \overline{\mathbf{N}}}{3 c^{2}} d S, \\
\mathbf{C}_{f s}=-\sum_{m_{\sigma}} \int_{S_{\sigma}} \mathbf{F}^{\mathrm{T}} \overline{\mathbf{N}} d S, \\
\Phi=\frac{\partial \mathbf{F}^{\mathrm{T}}}{\partial x} \frac{\partial \mathbf{F}}{\partial x}+\frac{\partial \mathbf{F}^{\mathrm{T}}}{\partial y} \frac{\partial \mathbf{F}}{\partial y}+\frac{\partial \mathbf{F}^{\mathrm{T}}}{\partial z} \frac{\partial \mathbf{F}}{\partial z} .
\end{gathered}
$$

Here $m_{f}, m_{\sigma}$ is the number of finite elements, which are used to decompose the fluid domain $V_{f}$ and the boundary $S_{\sigma}$; $\mathbf{f}$ is the vector of nodal values of the perturbation velocity potential $\phi ; \mathbf{F}, \overline{\mathbf{N}}$ are the matrices of the shape functions of the perturbation velocity potential and the normal component $w$ of the displacement vector of the plate.

Applying the known procedures of the finite element method to the variational equation (10) taking into account (6), (8), (9), we obtain the following matrix relation

$$
\begin{gathered}
\mathbf{M}_{s} \ddot{\mathbf{d}}+\mathbf{K}_{s} \mathbf{d}+\mathbf{C}_{s f} \dot{\mathbf{f}}+\mathbf{K}_{s f}^{v} \mathbf{f}=0, \\
\mathbf{M}_{s}=\sum_{m_{s}} \int_{V_{s}} \rho_{s} \mathbf{N}^{\mathrm{T}} \mathbf{N} d V, \mathbf{K}_{s}=\sum_{m_{s}} \int_{S_{s}} \mathbf{B}^{\mathrm{T}} \mathbf{D B} d S, \\
\mathbf{C}_{s f}=\sum_{m_{\sigma}} \int_{S_{\sigma}} \rho_{f} \mathbf{F}^{\mathrm{T}} \overline{\mathbf{N}} d S, \mathbf{K}_{s f}^{v}=\sum_{m_{\sigma}} \int_{S_{\sigma}} 2 \mu \mathbf{H} d S .
\end{gathered}
$$

Here, $m_{s}$ is the number of finite elements used to decompose the surface of the plate; $\mathbf{N}$ is the matrix of the shape function of the finite element, $\mathbf{B}$ is the matrix of the strainnodal displacement relationship, $\mathbf{H}$ is the matrix of the nodal displacement-velocity potential relationship.

The problem of studying the dynamic behavior of elastic plate interacting with a compressible viscous fluid is reduced to a simultaneous solution of the systems of equations (11) and (12). We consider the perturbed motion of the structure and fluid written as $(\mathbf{d}, \mathbf{f})=(\overline{\mathbf{d}}, \overline{\mathbf{f}}) \exp (\lambda t)$, 
where $\overline{\mathbf{d}}$ and $\overline{\mathbf{f}}$ are the vector functions of the coordinates, and $\lambda=\delta+i \omega$ the characteristic exponent. Here, $\omega$ and $\delta$ have the following physical meaning: $\omega$ is the natural frequency, and $\delta$ is damping. As a result, we obtain a generalized eigenvalue problem defined as

$$
\left[\begin{array}{cc}
\mathbf{C} & \mathbf{K}+\mathbf{A} \\
-\mathbf{I} & 0
\end{array}\right]\left\{\begin{array}{c}
\lambda \mathbf{x} \\
\mathbf{x}
\end{array}\right\}+\lambda\left[\begin{array}{cc}
\mathbf{M} & 0 \\
0 & \mathbf{I}
\end{array}\right]\left\{\begin{array}{c}
\lambda \mathbf{x} \\
\mathbf{x}
\end{array}\right\}=0
$$

where $\mathbf{I}$ is the unit matrix, $\mathbf{x}=\{\overline{\mathbf{d}}, \overline{\mathbf{f}}\}^{\mathrm{T}}$.

The complex eigenvalues of the coupled system of two equations (13) are calculated using an algorithm, which is based on the implicitly restarted Arnoldi method [8].

The model described above and the finite-element algorithm of its numerical implementation can easily be generalized to a configuration that is the structure comprising several parallel plates. Equation (10) is written for each elastic body, and expressions (1), (5), (7) - for each volume of the fluid. In the case of two plates, we obtain

$$
\begin{gathered}
\mathbf{M}\left\{\begin{array}{c}
\ddot{\mathbf{d}}_{1} \\
\ddot{\mathbf{f}} \\
\ddot{\mathbf{d}}_{2}
\end{array}\right\}+\mathbf{C}\left\{\begin{array}{c}
\dot{\mathbf{d}}_{1} \\
\dot{\mathbf{f}} \\
\dot{\mathbf{d}}_{2}
\end{array}\right\}+\mathbf{K}\left\{\begin{array}{c}
\mathbf{d}_{1} \\
\mathbf{f} \\
\mathbf{d}_{2}
\end{array}\right\}=0, \\
\mathbf{M}=\left[\begin{array}{ccc}
\mathbf{M}_{s_{1}} & 0 & 0 \\
\mathbf{M}_{f s_{1}}^{v} & \mathbf{M}_{f} & \mathbf{M}_{f s_{2}}^{v} \\
0 & 0 & \mathbf{M}_{s_{2}}
\end{array}\right], \mathbf{C}=\left[\begin{array}{ccc}
0 & \mathbf{C}_{s f_{1}} & 0 \\
\mathbf{C}_{f s_{1}} & \mathbf{C}_{f}^{v} & \mathbf{C}_{f s_{2}} \\
0 & \mathbf{C}_{s f_{2}} & 0
\end{array}\right], \\
\mathbf{K}=\left[\begin{array}{ccc}
\mathbf{K}_{s_{1}} & \mathbf{K}_{s f_{1}}^{v} & 0 \\
0 & \mathbf{K}_{f} & 0 \\
0 & \mathbf{K}_{s f_{2}}^{v} & \mathbf{K}_{s_{2}}
\end{array}\right] .
\end{gathered}
$$

Here the subindices «1» and «2» are used to identify variables belonging to the lower and upper plate, respectively. The eigenvalue problem will take the form of (13) up to the notation used.

\section{Experiment}

\subsection{Experiment setup}

The experimental setup is a demountable prismatic tank, the top cover (or part of it) of which is a plate of length $a$ and width $b$. Its thickness $h$ is an order of magnitude smaller than the thickness of the walls and the bottom of the container. The structure of the tank is shown in figure 1.

In the process of assembly, the tank is first filled with a fluid such that its level is slightly higher than the plate location. Then, a plate is dropped in special grooves cut in the side walls of the tank and additionally fixed by means of guide pins and demountable clamping frame. Under the weight of the plate, excess fluid is squeezed out through the technological holes or pumped out. This experimental setup provides the possibility for clamping or leaving free any of the four edges of the plate. This allows one to analyze various options for clamping, but requires the manufacture of test specimens, the dimensions of which correspond to the distance between the inner walls of the tank.

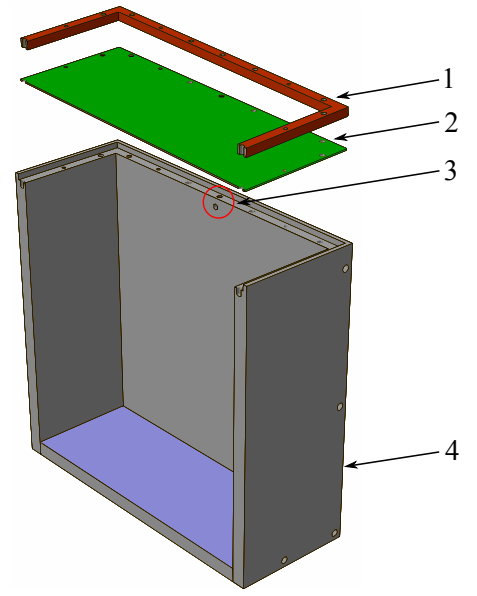

Figure 1. Experimental setup (in section): 1 - clamping frame; 2 - plate; 3 - technological hole; 4 - demountable tank

Table 1. Geometrical dimensions and boundary conditions for the plates.

\begin{tabular}{lllll}
\hline $\mathrm{N}$ & $h, \mathrm{~m}$ & $a, \mathrm{~m}$ & $b, \mathrm{~m}$ & $\mathrm{BC}$ \\
\hline 1 & $1.00 \times 10^{-3}$ & $20.00 \times 10^{-2}$ & $14.90 \times 10^{-2}$ & $\mathrm{CFCF}$ \\
2 & $7.08 \times 10^{-4}$ & $20.00 \times 10^{-2}$ & $28.15 \times 10^{-3}$ & $\mathrm{CFCF}$ \\
3 & $7.50 \times 10^{-4}$ & $14.10 \times 10^{-2}$ & $19.15 \times 10^{-3}$ & $\mathrm{CFFF}$ \\
\hline
\end{tabular}

Natural frequencies of the structure were investigated using a laser Doppler vibrometry method. As a source of excitation we used a speaker, which received a signal from computer's sound card. The duration of excitation was 12 seconds, during which the frequency varied from 1 to $2000 \mathrm{~Hz}$. The readings were taken by the Polytec PDV-100 digital laser vibrometer. The control point, at which the measurements were taken, was marked on the surface of the plate. The device was installed at a distance of $510 \mathrm{~mm}$ above the experimental setup and was oriented so that the angle between the laser ray and the surface of the plate was $90^{\circ}$. Such an arrangement made it possible to measure the normal component of the velocity vector $\dot{w}$. The natural vibration frequencies were determined from the Fourier transform analysis of the signal (corresponding to significant peaks). All test specimens were examined 30 times. Before each new measurement, the fluid was pumped out and the plate was demounted.

\subsection{Experiment results}

Geometrical parameters of the test plates are given in table 1. Here, $a$ and $b$ should be understood to mean dimensions that are not involved in clamping. In fact, the length and width of the samples is slightly larger. For the kinematic boundary conditions specified at the plate edges (BC) we introduce the following notation: $\mathrm{F}$ corresponds to the free edge and $\mathrm{C}-$ to rigid clamping. In designation of the specific boundary condition letter symbols are given in the clockwise direction starting from the left edge. For example, the variant CFFF corresponds to a cantilever plate clamped only at the left edge. 
Table 2. Experimentally obtained values of natural frequency $(\mathrm{Hz})$ of the plate interacting with air and fluid.

\begin{tabular}{cllllll}
\hline \multicolumn{2}{c}{ Sample } & $\omega_{1}$ & $\omega_{2}$ & $\omega_{3}$ & $\omega_{4}$ & $\omega_{5}$ \\
\hline 1 & Air & 230.4 & 257.1 & 359.3 & 591.5 & 667.0 \\
& & \pm 0.5 & \pm 0.5 & \pm 0.4 & \pm 0.3 & \pm 0.8 \\
\cline { 2 - 7 } & Fluid & 102.2 & 184.1 & 267.8 & 312.5 & 333.0 \\
& & \pm 0.5 & \pm 0.3 & \pm 0.1 & \pm 0.4 & \pm 0.3 \\
\hline 2 & Air & 92.5 & 255.0 & 417.8 & 502.4 & 833.1 \\
& & \pm 1.3 & \pm 2.2 & \pm 0.5 & \pm 3.5 & \pm 5.4 \\
\cline { 2 - 7 } & Fluid & 52.9 & 150.7 & 304.5 & 310.0 & 520.7 \\
& & \pm 10.9 & \pm 11.2 & \pm 11.9 & \pm 11.3 & \pm 13.3 \\
\hline 3 & Air & 32.3 & 202.7 & 463.1 & 568.7 & 1115.9 \\
& & \pm 0.2 & \pm 1.3 & \pm 0.7 & \pm 3.5 & \pm 6.6 \\
\hline & Fluid & 22.4 & 138.0 & 374.0 & 393.3 & 786.8 \\
& & \pm 0.7 & \pm 0.8 & \pm 1.5 & \pm 2.8 & \pm 5.2 \\
\hline
\end{tabular}

The average values of the five lowest natural frequencies of sample vibrations are given in table 2 . In each cell of the table the numbers in the second line show the value of the mean square deviation $s$, obtained from 30 measurements:

$$
s=\sqrt{\frac{\sum_{i=1}^{30}\left(\omega_{n}-\omega\right)^{2}}{n-1}},
$$

where $\omega$ is the arithmetic mean value.

The results demonstrate that when the plate interacts with a fluid, the frequency spectrum is significantly reduced. The frequency $\omega_{1}$ of each of the test specimens decreases by $55.6 \%, 42.8 \%$ and $30.6 \%$, respectively. Note that the maximum difference corresponds to the plate with the largest contact area (specimen 1).

\section{Numerical results}

Verification of the developed finite-element algorithm was carried out by comparing the calculated natural oscillation frequencies with the experimental values given in Section 4.2. The calculations were made using the following characteristics: $E=201 \mathrm{GPa}, \rho_{s}=7850 \mathrm{~kg} / \mathrm{m}^{3}, v=0.30$, $\rho_{f}=1000 \mathrm{~kg} / \mathrm{m}^{3}, \mu=8.94 \times 10^{-4} \mathrm{~Pa} \mathrm{~s}, c=1500 \mathrm{~m} / \mathrm{s}$. The obtained results are presented in table 3 . The numbers in the second line in each cell of the table indicate the relative error compared to the experimental data.

The computational scheme applied for samples 2 and 3 did not take into account the gravitational waves excited at the free surface of the fluid under vibration. The corresponding boundary condition used in the model is represent as $\phi=0$. Despite such an assumption, the obtained numerical results agree with the experimental data within the relative accuracy of $6 \%$.

To reveal new relationships determining the dynamic characteristics of plates interacting with a viscous fluid, a series of computational experiments was carried out. The calculations were performed for the following geometric dimensions and physicomechanical characteristics: $a=0.24 \mathrm{~m}, b=0.32 \mathrm{~m}, h=2 \times 10^{-3} \mathrm{~m}, H=4 \times 10^{-2} \mathrm{~m}$, $E=69 \mathrm{GPa}, v=0.30, \rho_{s}=2700 \mathrm{~kg} / \mathrm{m}^{3}, \rho_{f}=1000$ $\mathrm{kg} / \mathrm{m}^{3}, \mu=8.94 \times 10^{-4}$ Pa s, $c=1500 \mathrm{~m} / \mathrm{s}$.
Table 3. Comparison of natural frequencies $(\mathrm{Hz})$ of plates interacting with air and fluid.

\begin{tabular}{|c|c|c|c|c|c|c|}
\hline \multicolumn{2}{|c|}{ Sample } & $\omega_{1}$ & $\omega_{2}$ & $\omega_{3}$ & $\omega_{4}$ & $\omega_{5}$ \\
\hline \multirow[t]{4}{*}{1} & Air & 243.7 & 270.5 & 373.9 & 587.0 & 672.1 \\
\hline & & $-5.8 \%$ & $-5.2 \%$ & $-4.1 \%$ & $0.8 \%$ & $-0.8 \%$ \\
\hline & Fluid & 97.8 & 174.3 & 288.8 & 313.5 & 327.7 \\
\hline & & $4.3 \%$ & $5.3 \%$ & $-8.6 \%$ & $-0.3 \%$ & $1.6 \%$ \\
\hline \multirow[t]{4}{*}{2} & Air & 93.6 & 257.9 & 422.6 & 506.0 & 837.9 \\
\hline & & $-1.2 \%$ & $-1.1 \%$ & $-1.2 \%$ & $-0.7 \%$ & $-0.6 \%$ \\
\hline & Fluid & 55.0 & 155.7 & 313.4 & 325.5 & 533.4 \\
\hline & & $-3.9 \%$ & $-3.3 \%$ & $-2.9 \%$ & $-5.0 \%$ & $-2.4 \%$ \\
\hline \multirow[t]{4}{*}{3} & Air & 31.6 & 197.6 & 448.5 & 553.8 & 1087.3 \\
\hline & & $2.2 \%$ & $2.5 \%$ & $3.1 \%$ & $2.6 \%$ & $2.6 \%$ \\
\hline & Fluid & 22.4 & 140.9 & 383.2 & 400.2 & 788.3 \\
\hline & & $0.1 \%$ & $-2.1 \%$ & $-2.4 \%$ & $-1.7 \%$ & $-0.2 \%$ \\
\hline
\end{tabular}

The investigation of the natural vibrations of a plate interacting with an ideal fluid was carried out in works $[9,10]$. The results obtained with a new model confirm the well-known fact that viscosity of a liquid has practically no effect on the vibrational frequency. In this regard, the curves shown in figure $2 \mathrm{a}$, a completely coincide with those published in [9]. The dependence of the lowest natural frequency of a single plate on the height of the fluid layer is asymptotic if the ratio $H / b>0.75$. However, for a parameter $\delta$, characterizing damping of the system, such a tendency manifests itself to a lesser extent and is determined by clamping conditions (see figure $2 \mathrm{~b}$ ).

The analysis of two parallel plates showed that the dependence of any fixed frequency in the spectrum and its damping on the ratio $H / b$ is strongly nonmonotonic in nature and may have kinks due to a change in the vibration modes [10]. To illustrate this fact, we present figure 3a, which shows that the two lowest vibration modes $(1,0)$ and $(0,0)$ correspond to the lowest natural frequency $\omega_{1}$ in the examined interval $H / b$. Here, $(n, m)$ denotes the number of nodal lines in the direction of the $O x-$ and $O y-$ axes. Figure $3 \mathrm{~b}$ demonstrates the response of the parameter $\delta$ to a change of the vibration mode in the vicinity of point $H / b \approx 0.265$. It is seen that at this instant of time an abrupt change of damping occurs.

\section{Conclusions}

Using the developed test setup, we carried out an experimental study of the vibration frequencies of a plate in contact with a fluid for different types of fixing.

For the developed mathematical formulation of the problem of spatial vibrations of plates interacting with a viscous fluid, we constructed a finite-element algorithm. Its reliability was verified by comparing the obtained results with experimental data. The calculations made it possible to conclude for two parallel plates that the dependence of any fixed frequency in the spectrum and its damping on the height of the liquid layer is of strongly pronounced nonmonotonic character and may have kinks due to a change in vibration mode.

The work is supported by the Russian Foundation for Basic Research grant (project N 16-41-590646). 

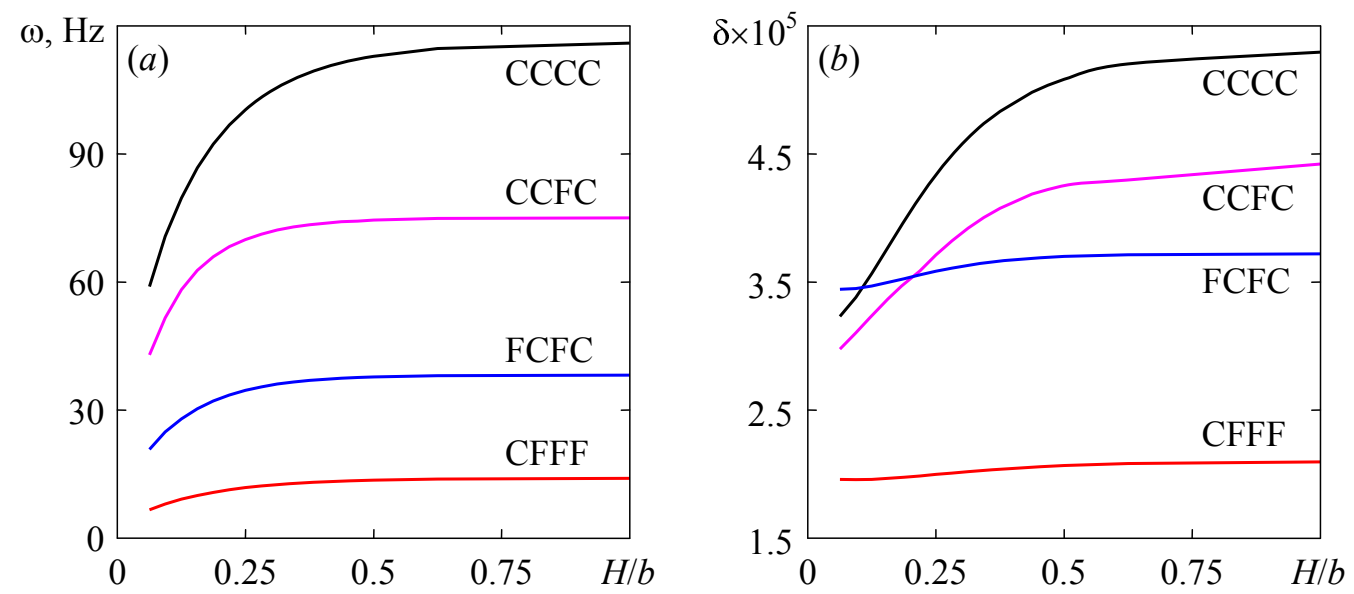

Figure 2. Plots of lowest natural frequencies of the vibration $\omega$ (a) and damping $\delta$ (b) versus the height of the fluid layer $H$ for the plate at different variants of boundary conditions
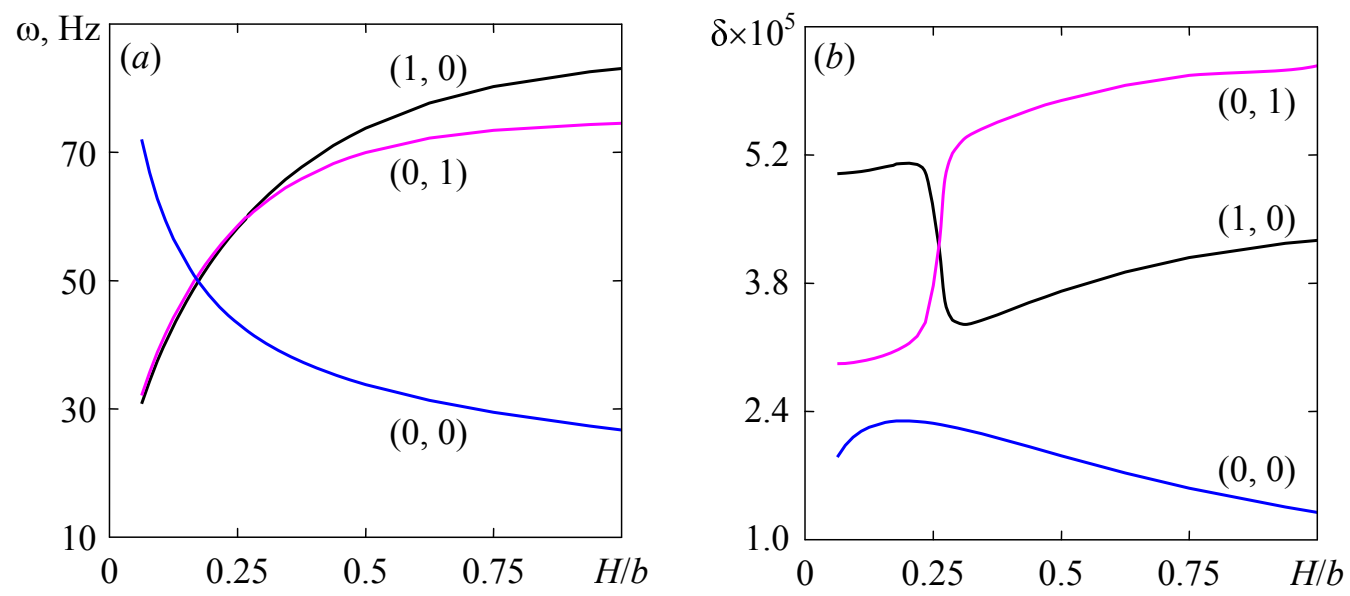

Figure 3. Natural frequencies of vibration $\omega$ (a) and damping $\delta$ (b) versus the height of the fluid layer (CCFC), for two plates

\section{References}

[1] X. Huang, C. Valero, E. Egusquiza, A. Presas, A. Guardo. Computers \& Fluids. 71, 54-64 (2013)

[2] E. Askari, K.-H. Jeong, M. Amabili. J. Sound Vib. 332, 3064-3085 (2013)

[3] U.S. Lindholm, D.D. Kana, W.H. Chu et al. J. Ship Res. 9, 11-22 (1965)

[4] M.R. Haddara, S. Cao. Mar. Struct. 9, 913-933 (1996)

[5] C.-Y. Liao, Y.-C. Wu, C.-Y. Chang, C.-C. Ma. J. Sound Vib. 394, 545-574 (2017)
[6] D.D. Joseph. J. Fluid Mech. 479, 191-197 (2003)

[7] J.N. Reddy. An introduction to nonlinear finite element analysis, 2nd edn. (Oxford University Press, Oxford, UK, 2015)

[8] R.B. Lehoucq, D.C. Sorensen. SIAM J. Matrix Anal. Appl. 17, 789-821 (1996)

[9] S.A. Bochkarev, S.V. Lekomtsev, V.P. Matveenko. Fluid Dyn. 51, 821-833 (2016)

[10] S.A. Bochkarev, S.V. Lekomtsev. Solid State Phenomena. 243, 51-58 (2016) 